\title{
Offset-free nonlinear Model Predictive Control with state-space process models
}

\author{
PIOTR TATJEWSKI
}

\begin{abstract}
Offset-free model predictive control (MPC) algorithms for nonlinear state-space process models, with modeling errors and under asymptotically constant external disturbances, is the subject of the paper. The main result of the paper is the presentation of a novel technique based on constant state disturbance prediction. It was introduced originally by the author for linear state-space models and is generalized to the nonlinear case in the paper. First the case with measured state is considered, in this case the technique allows to avoid disturbance estimation at all. For the cases with process outputs measured only and thus the necessity of state estimation, the technique allows the process state estimation only - as opposed to conventional approach of extended process-and-disturbance state estimation. This leads to simpler design with state observer/filter of lower order and, moreover, without the need of a decision of disturbance placement in the model (under certain restrictions), as in the conventional approach. A theoretical analysis of the proposed algorithm is provided, under applicability conditions which are weaker than in the conventional approach. The presented theory is illustrated by simulation results of nonlinear processes, showing competitiveness of the proposed algorithms.
\end{abstract}

Key words: nonlinear control, predictive control, offset-free control, state-space model, state estimation.

\section{Introduction}

The MPC is now a well established advanced control technology, represented by a variety of successful control algorithms and software packages applied in practice, see, e.g., [5], [9], [16], [19], [3], [20], [28], [18], [21], [22], [4], [25]. First MPC algorithms used linear nonparametric models in the form of impulse or step responses (e.g., a well known DMC algorithm). Then transfer function models were used (GPC algorithm). Relatively later, the state-space modeling was applied in MPC structures leading to a variety of successful algorithms, including those with nonlinear models. There are different approaches to state-space modeling, as minimal and non-minimal models, extended velocity form models - leading to different MPC designs. From practical point of view, the offset-free property is necessary for any MPC algorithm, especially for process control

The Author is with Warsaw University of Technology, Nowowiejska 15/19, 00-665 Warszawa, Poland, e-mail: P.Tatjewski@ia.pw.edu.pl

Received 14.10.2017. 
applications. This means that constant, or asymptotically constant disturbances should be attenuated by the algorithm without steady-state error (the offset). It should be also realized that points and ways these disturbances influence the process are also important for the MPC design. The mentioned problem has attracted a rather limited attention in the literature, until the last decade [13], [15], [20], [14], [6], [11], [12], [23], [8]. However, there is still a certain lack of a clear understanding how the mentioned disturbances (which include modeling errors) can be most effectively treated in the MPC algorithms with state-space models. We concentrate on this problem in the paper, for the defined class of asymptotically constant deterministic disturbances. More general, continuously varying disturbances, like sinusoids, will be not considered, see [11] for offset-free MPC reference tracking under varying disturbances.

The main subject of the paper is to present new technique of offset-free MPC design for nonlinear processes, under (asymptotically) constant set-point values and (asymptotically) constant disturbances entering the process at any point, possibly with white noises added, and to compare this technique with the existing ones. The considered class of disturbances, important e.g. in process control, includes modeling errors and external step disturbances or piecewise-constant disturbances changing rarely with respect to the controlled process dynamics. Main contribution of the paper is to present new techniques for both measured and estimated process state, in the latter case with estimation of the process state only. The proposed approach generalizes the results obtained earlier by the author for linear models [20], [23], to the case of nonlinear state-space process models. This leads to simpler and more general MPC structures than the previous conventional approach. The paper is a revised and extended version of the conference papers [24], [26].

The structure of the paper is as follows. In Section 2 the MPC is briefly reviewed, to introduce formulations needed for further considerations. In Section 3 the case with measured state is presented, resulting in a formulation of the MPC algorithm where the observer/estimator of the considered deterministic disturbances is not needed at all. In Section 4 the most general case with state estimation is treated. The results presented in these two main sections lead to simpler nonlinear MPC control structures and simpler design as well, under significantly weaker applicability conditions. In Section 5, the proposed MPC approach is illustrated by simulations of the control structure with nonlinear example process models. Finally, conclusions are formulated.

\section{Predictive Control briefly recalled}

The principle of MPC is now well known, different formulations of MPC algorithms can be found in many papers and books. In this paper, we are interested in discrete-time state-space process models. In the books, see [9], [19], [20], [28], [18], mainly linear process models, of different types, are considered. Nonlinear process models used in the MPC algorithms can be in the form of nonlinear state-space models or nonlinear difference equations of higher orders, including neural network models, see, e.g., [27], [7]. 
In this section, we shall briefly recall the MPC formulation needed for further presentation in the paper. The principle of MPC is to evaluate the current process control input signal by minimizing, at each sampling instant $k$, a performance function (cost function) over a future prediction horizon of $N$ samples. The following performance function is one of the most widely used in process control:

$$
J(k)=\sum_{p=1}^{N} \|\left[y^{s p}(k+p \mid k)-y(k+p \mid k)\left\|_{\Psi}^{2}+\sum_{p=0}^{N_{u}-1}\right\| \Delta u(k+p \mid k) \|_{\Lambda}^{2},\right.
$$

where $\|x\|_{\boldsymbol{R}}^{2}=x^{T} \boldsymbol{R} x, \boldsymbol{\Psi} \geqslant \mathbf{0}$ and $\boldsymbol{\Lambda}>\mathbf{0}$, are square diagonal scaling matrices of dimensions corresponding to the dimensions $n_{y}$ and $n_{u}$ of the process controlled output and control input vectors, respectively (a simpler formulation of (1) is often used in theoretical considerations, with one scaling scalar $\lambda$ only, i.e., $\boldsymbol{\Psi}=\boldsymbol{I}$ and $\boldsymbol{\Lambda}=\boldsymbol{\lambda} \boldsymbol{I})$. In the formulation (1), $N_{u} \leqslant N$ denotes the length of the control horizon, $y^{s p}(k+p \mid k)$ and $y(k+p \mid k)$ are set-point (reference) and process output vectors predicted for a future sample $k+p$, but calculated at the current sample $k, p=1, \ldots, N$. The control inputs on the control horizon are the decision variables, whereas $\triangle u(k+p \mid k)=u(k+p \mid k)-u(k+p-1 \mid k)$, $p=0, \ldots, N_{u}-1$. The vector of the decision variables will be denoted by $U(k)$,

$$
U(k)=\left[u(k \mid k)^{T} u(k+1 \mid k)^{T} \cdots u\left(k+N_{u}-1 \mid k\right)^{T}\right]^{T} .
$$

We assume that the optimization of $J(k)$ is subject to simple constraints:

$$
\begin{aligned}
-\triangle u_{\max } & \leqslant \triangle u(k+p \mid k) \leqslant \triangle u_{\max }, p=0, \ldots, N_{u}-1, \\
u_{\min } & \leqslant u(k+p \mid k) \leqslant u_{\max }, p=0, \ldots, N_{u}-1, \\
y_{\min } & \leqslant y(k+p \mid k) \leqslant y_{\max }, p=1, \ldots, N .
\end{aligned}
$$

More general form of the constraints, including any linear functions of all variables used, is possible, but avoided here for simplicity.

Denoting composite vectors of set-points and predicted outputs on the prediction horizon by $Y^{s p}(k)$ and $Y^{p r}(k)$, respectively,

$$
\begin{gathered}
Y^{s p}(k)=\left[\begin{array}{lll}
y^{s p}(k+1 \mid k)^{T} & \ldots & y^{s p}(k+N \mid k)^{T}
\end{array}\right]^{T}, \\
Y^{p r}(k)=\left[\begin{array}{lll}
y(k+1 \mid k)^{T} & \ldots & y(k+N \mid k)^{T}
\end{array}\right]^{T},
\end{gathered}
$$

we can formulate, in a compact form, the MPC optimization problem which calculates the optimal control trajectory:

$$
\begin{aligned}
\min _{U(k)}\{J(k)= & \left.\left\|Y^{s p}(k)-Y^{p r}(k)\right\|_{\underline{\Psi}}^{2}+\|\triangle U(k)\|_{\underline{\Lambda}}^{2}\right\} \\
& \text { subject to (3), (4) and (5), }
\end{aligned}
$$

where

$$
\underline{\boldsymbol{\Psi}}=\operatorname{diag}\{\overbrace{\boldsymbol{\Psi}, \ldots, \boldsymbol{\Psi}}^{N \text { times }}\}, \quad \underline{\boldsymbol{\Lambda}}=\operatorname{diag}\{\overbrace{\boldsymbol{\Lambda}, \ldots, \boldsymbol{\Lambda}}^{N_{u} \text { times }}\},
$$


and the predicted output trajectory $Y^{\operatorname{pr}}(k)$ is calculated using the process model. When this model is nonlinear, then the optimization problem (8) is also nonlinear and nonlinear optimization procedures must be applied.

With a linear process model, the MPC optimization problem (8) is a strictly convex quadratic programming $(\mathrm{QP})$ problem, thus with a well defined, unique solution provided the set defined by the constraints assures feasibility (is non-empty). For a detailed description of the MPC algorithms with linear process models, see, e.g., [20]. For nonlinear models, uniqueness of solution points cannot be guaranteed.

Once the MPC optimization problem has been solved, the first element $u(k \mid k)=u(k)$ of the control trajectory is used only and applied as the process input. After the next measurement (at the next sampling instant) the whole procedure is repeated (receding horizon strategy).

We shall assume $n_{u}=n_{y}$ in the paper, which usually (in the linear case always) yields a unique solution of the MPC optimization problem. Nevertheless, the case $n_{u}>n_{y}$, not unusual in MPC applications, will be commented.

\section{Offset-free nonlinear MPC with measured state}

The following description will be used to represent the nonlinear process:

$$
\begin{aligned}
x(k+1) & =f_{p}\left(x(k), u(k), d_{p}(k)\right), \\
y(k) & =g(x(k)),
\end{aligned}
$$

where $x$ denotes the process state vector, $\operatorname{dim} x=n_{x}, y$ the controlled output vector, $\operatorname{dim} y=n_{y}, u$ the control (control input) vector, $\operatorname{dim} u=n_{u}$ and $d_{p}$ represents unknown, unmeasured disturbances (including modeling errors), $\operatorname{dim} d_{p}=n_{d_{p}}$. Measured disturbances will not be explicitly considered in the paper, for the sake of simplicity.

The process model in the following form will be assumed to be known:

$$
\begin{aligned}
x(k+1) & =f(x(k), u(k)), \\
y(k) & =g(x(k)) .
\end{aligned}
$$

But for state prediction in the MPC algorithm, (11) will be augmented to the form

$$
\begin{aligned}
x(k+1) & =f(x(k), u(k))+v(k), \\
y(k) & =g(x(k)),
\end{aligned}
$$

where $v(k)$ will represent influence of unmeasured disturbances on the state vector, $\operatorname{dim} v=n_{x}[20,23]$. The key factor of the presented MPC algorithm is the way $v(k)$ is modeled for the use in (12a). The constant state disturbance prediction, originally proposed in [20] for the MPC with linear state-space process models (see also [23]), can be generalized to the nonlinear case, as follows

$$
v(k)=x(k)-f(x(k-1), u(k-1)),
$$




$$
v(k \mid k)=v(k+1 \mid k)=v(k+2 \mid k)=\cdots=v(k+N-1 \mid k)=v(k),
$$

where $x(k)$ denotes the process state measured at time $k$.

The nonlinear MPC algorithm solves, at each sampling instant $k$, the MPC optimization problem (8), using a nonlinear optimization procedure with the vector of decision variables $U(k)$ defined by (2). The procedure iterates the decision variables, starting from an initial vector $U^{(0)}(k)$ and calculating subsequent (improved) values $U^{(i)}(k), i=1,2,3, \ldots$, etc., until certain optimality criterion is fulfilled. For each new value $U^{(i)}(k)$ calculation of the output predictions $y^{(i)}(k+p \mid k)$ over the control horizon (i.e., for $p=1, \ldots, N$ ) is needed.

The nonlinear MPC algorithm with measured process state, called further the Algorithm NMPC1, is now presented, in the form of steps performed at each sampling instant $k$ :

\section{Algorithm NMPC1}

1. The process state $x(k)$ is measured.

2. The state disturbance prediction vector $v(k)$ is calculated according to (13).

3. The optimization problem (8) is solved, by an iterative nonlinear optimization procedure, calculating at its every $(i-$ th) internal iteration the output predictions as follows:

3a. Using the model (11a), state predictions $x^{(i)}(k+p \mid k)$ are recursively calculated over the control horizon, i.e., for $p=1, \ldots, N$ :

$$
x^{(i)}(k+p+1 \mid k)=f\left(x^{(i)}(k+p \mid k), u^{(i)}(k+p \mid k)\right)+v(k),
$$

where $x^{(i)}(k \mid k)=x(k)$ is the measured state at the current sample, $\left.u^{(i)}(k+p \mid k)\right)$ are elements of the vector of decision variables (2) and

$$
u^{(i)}(k+p \mid k)=u^{(i)}\left(k+N_{u}-1 \mid k\right) \text { for } p \geqslant N_{u} .
$$

3b. The output predictions are calculated:

$$
y^{(i)}(k+p \mid k)=g\left(x^{(i)}(k+p \mid k)\right), \quad p=1, \ldots, N .
$$

4. After the optimal trajectory $\hat{U}(k)$ is found by the optimization procedure, its first element $\hat{u}(k \mid k)$ is the current control input signal $u(k)$, which is sent to the process actuators.

Before stating a theorem about offset-free property of the NMPC1 algorithm, we shall formulate assumptions about the class of disturbances and about the set-points.

Assumption 1 The disturbances affecting the process (10a)-(10b) are asymptotically constant, stabilizing at a certain value $d_{s s}$, the set-point (reference) values are asymptotically constant, stabilizing at $y_{\text {ss }}^{s p}$. 
Assumption 2 The set-point values $y_{s s}^{s p}$ are feasible and steady-state controllable for disturbance values $d_{s s}$, i.e., $y_{s s}^{s p}$ satisfies the output constraints (5) and there is a feasible control signal $u$ (i.e., satisfying (4)) resulting in the process output $y(u)=y_{s s}^{s p}$ in steady-state, under the disturbance values $d_{s s}$.

Assumption 1 of defines the class of disturbance and set-point functions under consideration. Assumption 2 is natural, the steady-state value of the set-point must be feasible and steady-state controllable (attainable) to make the offset-free control possible, regardless of a kind of the feedback controller used. We shall discuss this assumption in greater detail after Theorem 7, which formulates conditions for offset-free property of the feedback control system with the Algorithm NMPC1.

\section{Theorem 7 Assume that}

- Assumptions 1 and 2 are satisfied,

- Assumption 3: the MPC optimization problem (8) with output predictions as defined by (15)-(17) is feasible for every $k$ (i.e., the set defined by the constraints (3)-(5) is not empty) and the feedback control system consisting of the process (10a)-(10b) and the Algorithm NMPC1 is asymptotically stable,

then the feedback control system defined in Assumption 3 provides offset-free control, i.e., the process outputs stabilize at the set-point values $y_{s s}^{s p}$, despite the influence of all the disturbances as defined in Assumption 1.

Proof. Consider state prediction equations (15) (with the iteration superscript omitted), together with the state disturbance predictions (13):

$$
\begin{gathered}
x(k+1 \mid k)=f(x(k), u(k \mid k))+[x(k)-f(x(k-1), u(k-1))], \\
x(k+2 \mid k)=f(x(k+1 \mid k), u(k+1 \mid k))+[x(k)-f(x(k-1), u(k-1))], \\
\vdots \\
x\left(k+N_{u} \mid k\right)=f\left(x\left(k+N_{u}-1 \mid k\right), u\left(k+N_{u}-1 \mid k\right)\right)+[x(k)-f(x(k-1), u(k-1))], \\
x\left(k+N_{u}+1 \mid k\right)=f\left(x\left(k+N_{u} \mid k\right), u\left(k+N_{u}-1 \mid k\right)\right)+[x(k)-f(x(k-1), u(k-1))], \\
\vdots \\
x(k+N \mid k)=f\left(x(k+N-1 \mid k), u\left(k+N_{u}-1 \mid k\right)\right)+[x(k)-f(x(k-1), u(k-1))] .
\end{gathered}
$$

As the set-point signal is asymptotically constant, $y^{s p}(k) \rightarrow y_{s s}^{s p}$, the disturbances are asymptotically constant and the control system is asymptotically stable, then at the steady-state the process state $x(k)$, the outputs $y(k)$ and the inputs $u(k)$ stabilize at certain constant values $x_{s s}, y_{s s}=g\left(x_{s s}\right)$ and $u_{s s}$, respectively.

As the process state stabilizes at the value $x_{s s}$ and the corresponding control vector at the value $u_{s s}$, then examining, recursively, the state prediction equations (18a)-(18e), we can conclude that at the steady-state: 
- it follows from (18a) that $x(k+1 \mid k)$ stabilizes at $x_{s s}$,

- the above and (18b) implies that $x(k+2 \mid k)$ stabilizes at $x_{s s}$,

- etc., until $x(k+N \mid k)$ stabilizes at $x_{s s}-$ i.e., all state predictions $x(k+p \mid k)$ on the prediction horizon stabilize at $x_{s s}$.

Taking now into account this result and the output prediction equation (17), we conclude that the predicted outputs stabilize, at the steady-state, at the value:

$$
y(k+p \mid k)=g\left(x_{s s}\right)=y_{s s}, \quad p=1, \ldots, N .
$$

It remains to prove that

$$
y(k+p \mid k)=y_{s s}=y_{s s}^{s p}, \quad p=1, \ldots, N,
$$

that this, the performance function (1) stabilizes at its most minimal, zero value. This follows from the form of this function, because:

- at the steady-state the control increments must be zero,

- the equality (20) must be satisfied, provided the set-point value $y_{s s}^{s p}$ is attainable (feasible and steady state controllable, Assumption (2)). If (20) was not true, then the first sum in the performance function (1) would be greater than zero and, at the same time, there would be freedom to change the control signal by the optimization procedure to force (1) towards the lower, zero value - as $y_{s s}^{s p}$ attainable. This would contradict the fact that (1) is minimized at each sampling instant $k$ (the MPC optimization problem (8) is feasible for every $k$, Assumption (3)).

This completes the proof.

Given the process equations (10a)-(10b), certain analysis of steady-state controllability conditions stated in Assumption 2 can be performed using the Implicit Function Theorem. The following algebraic equations are the process equations at steady state:

$$
\begin{aligned}
& x_{s s}=f_{p}\left(x_{s s}, u_{s s}, d_{s s}\right), \\
& y_{s s}=g\left(x_{s s}\right) .
\end{aligned}
$$

For steady-state controllability, we are looking for a solution of this set of equations with respect to $\left(x_{s s}, u_{s s}\right)$, for given values of $\left(y_{s s}, d_{s s}\right)$. From elementary algebra, a necessary requirement for such a solution is $n_{u} \geqslant n_{y}$ (excluding degenerate cases). Assume the functions $f_{p}$ and $g$ are continuously differentiable on their domains and define

$$
\begin{aligned}
& \boldsymbol{F}_{x}=\frac{\partial}{\partial x} f_{p}\left(x_{s s}, u_{s s}, d_{s s}\right), \\
& \boldsymbol{F}_{u}=\frac{\partial}{\partial u} f_{p}\left(x_{s s}, u_{s s}, d_{s s}\right), \\
& \boldsymbol{G}_{x}=\frac{\partial}{\partial x} g\left(x_{s s}\right) .
\end{aligned}
$$


Denoting the system of equations (21a)-(21b) as a nonlinear mapping

$$
\mathcal{H}\left(x_{s s}, u_{s s}, d_{s s}, y_{s s}\right)=0,
$$

the Jacobian $\boldsymbol{J}_{x u}$ of this mapping, with respect to $\left(x_{s s}, u_{s s}\right)$, is

$$
\boldsymbol{J}_{x u}=\left[\begin{array}{cc}
\boldsymbol{I}-\boldsymbol{F}_{x} & -\boldsymbol{F}_{u} \\
-\boldsymbol{G}_{x} & \mathbf{0}
\end{array}\right]
$$

If $n_{u}=n_{y}$, which has been assumed for the performance function (1), then the Jacobian $\boldsymbol{J}_{x u}$ is a square matrix. Now, it follows from the Implicit Function Theorem, that if $\operatorname{det} \boldsymbol{J}_{x u}\left(x_{s s}, u_{s s}, d_{s s}, y_{s s}\right) \neq 0$, then there exists a neighborhood of $\left(y_{s s}, d_{s s}\right)$, where the system of equations (21a)-(21b) has a unique solution with respect to $\left(x_{s s}, u_{s s}\right)$, i.e., we have a unique steady-state controllability. Certainly, in a practical application the Jacobian should be nonsingular for all values of its arguments from a working region of the control system, i.e., corresponding to all possible values of $y_{s s}$ and $d_{s s}$.

The case $n_{u}>n_{y}$ is not uncommon in MPC applications, having a potential to select more effective control inputs. However, the set-point information must than include also desired steady-state values $u_{s s}^{s p}$ of the process control inputs, corresponding to the values $y_{s s}^{s p}$ (provided by a local steady-state optimization or a steady-state target optimization, see, e.g., [16], [21]). The performance function (1) should be than modified, by adding additional, regularizing term

$$
\sum_{p=0}^{N_{u}-1}\left\|u(k+p \mid k)-u_{s s}^{s p}\right\|_{\boldsymbol{\Lambda}_{u}}^{2},
$$

where $\boldsymbol{\Lambda}_{u}$ is a weighting matrix, or even using (27) instead of the second sum in (1) [16], [21]. The algorithm NMPC1 can also be used in this case, providing offset-free output control under the assumptions of Theorem 7.

On the other hand, the control system can work properly even if steady-state feasibility does not hold in Assumption 2 of Theorem 1, possibly violated due to active output constraints (5). Then the offset-free control is certainly not possible. However, there are well known measures to assure MPC optimization feasibility in this case, usually by a suitable reformulation of the MPC optimization problem, using softened versions of the output constraints instead of hard ones (5), see, e.g., [16], [20]. What necessarily must be then fulfilled, is the Assumption (2) restricted to steady-state controllability under input feasibility only (i.e., existence of $u_{s s}$ satisfying the hard constraints (3)-(4)). In the considered case, the process outputs would stabilize at a value closest to $y_{s s}^{s p}$, in the least-squares sense. The other possibility is to solve first, at each sampling instant, a static optimization problem to find a steady-state feasible target $y_{s s}^{t}$ - the output value which is feasible and closest to the set-point $y_{s s}^{s p}$ (a steady-state target optimization, see, e.g., [17], [16], [20], [12]). Then this target is then used in the MPC optimization problem (8), in place of the set-point. 
Assumption 3 of Theorem 8 is important for a proper action of the MPC controller, as infeasibility of the MPC optimization problem can be caused by the output constraints. It must be avoided in on-line MPC control, and there are well known measures to do that, as the one just discussed above (the use of soft formulations of the output constraints). The asymptotic stability of the control system is a basic requirement, widely discussed in the literature - its analysis is not the aim of this paper, directed towards the design of controller structures assuring offset-free output control.

The essence and novelty of the algorithm NMPC1 is that the controller assures the offset-free control without the necessity to use an observer/estimator of the deterministic disturbances. It should be pointed out that the key factor which makes that the algorithm works is the use of the state disturbance prediction $v(k)$ defined by (13)-(14) and used in prediction equations (15). This results in a compensation of state modeling errors in steady-states, which can be easily seen from the following equations:

$$
v_{s s}=x_{s s}-f\left(x_{s s}, u_{s s}\right)=f_{p}\left(x_{s s}, u_{s s}, d_{s s}\right)-f\left(x_{s s}, u_{s s}\right) .
$$

This means that, in fact, any process model may be used, from the point of view of the offset-free control property only, provided the overall feedback control loop is asymptotically stable. Certainly, this is not the whole truth, as quality of the model influences the feedback loop stability properties, quality of dynamic state and output predictions over the prediction horizon, and thus the quality of the dynamic properties of the MPC controller.

Notice that $g(x)$ has been assumed to be a known nonlinear function of the state. In practice it is always true (and in most cases $g(x)$ is linear). However, if it would not be true, a simple output disturbance model must be added in the algorithm to assure offset-free control, in the same way as in the case with linear process model, see [23].

\section{Offset-free nonlinear MPC with state estimation}

\subsection{The technique of extended state estimation}

When the process state needs estimation, a conventional technique for offset-free control in the linear model case is the addition of a disturbance state model and the extended state (process-and-disturbance state) estimation, see [13], [15], [6], [10], [23]. The analysis is here made usually applying deterministic state observers, as it is a simpler approach and all results can be easily generalized to the case with Kalman filtering. A restrictive applicability condition of this approach is the requirement that the number of disturbances located in the model must not exceed the number of process measured outputs, which leads to the necessity of a thorough choice of the place where these disturbances are located in the model.

An extension of this conventional approach to the nonlinear case can be found, e.g., in [12]. The augmented process model is introduced, in the form 


$$
\begin{aligned}
x(k+1) & =f_{\text {aug }}(x(k), u(k), d(k)), \\
d(k+1) & =d(k), \\
y(k) & =g_{\text {aug }}(x(k), d(k)),
\end{aligned}
$$

where $d(k), \operatorname{dim} d=n_{d}$, represents the state of disturbances appropriately located in the process model. The Extended Luenberger Observer (ELO) is then applied to the extended state estimation [12]:

$$
\begin{aligned}
& \hat{x}(k)=f_{\text {aug }}(\hat{x}(k-1),u(k-1), \hat{d}(k-1))+ \\
&+\boldsymbol{L}_{x}\left[y(k-1)-g_{\text {aug }}(\hat{x}(k-1), \hat{d}(k-1))\right], \\
& \hat{d}(k)=\hat{d}(k-1)+\boldsymbol{L}_{d}\left[y(k-1)-g_{\text {aug }}(\hat{x}(k-1), \hat{d}(k-1))\right],
\end{aligned}
$$

where $\hat{x}(k)$ and $\hat{d}(k)$ represent estimates of the process state and disturbance state, respectively. $\boldsymbol{L}_{x}$ and $\boldsymbol{L}_{d}$ are gain matrices of the observer, usually designed as for the standard observer for a linear process model (linearization of (29) at the working point). Adaptation of the gain matrices can be also applied.

The disadvantage of the observer (30) is that it applies delayed input measurement, it is a predictive observer (by analogy with the linear case [1]). More practical is the the current observer:

$$
\begin{aligned}
\hat{x}(k \mid k-1) & =f_{\text {aug }}(\hat{x}(k-1), u(k-1), \hat{d}(k-1)), \\
\hat{d}(k \mid k-1) & =\hat{d}(k-1), \\
\hat{x}(k) & =\hat{x}(k \mid k-1)+\boldsymbol{L}_{c x}\left[y(k)-g_{\text {aug }}(\hat{x}(k \mid k-1), \hat{d}(k \mid k-1))\right], \\
\hat{d}(k) & =\hat{d}(k \mid k-1)+\boldsymbol{L}_{c d}\left[y(k)-g_{\text {aug }}(\hat{x}(k \mid k-1), \hat{d}(k \mid k-1))\right],
\end{aligned}
$$

where $\boldsymbol{L}_{c x}$ and $\boldsymbol{L}_{c d}$ are gain matrices of the observer. The Extended Kalman Filter (EKF) can be applied in place of the ELO observer as well.

The MPC controller designed according to this more conventional technique of extended process-and-disturbance state does not use the disturbance model (13)-(14), instead the modeled disturbance estimate $\hat{d}(k)$ is appropriately used in state and output predictions. The disadvantage of this technique, both in the linear and, especially, in the nonlinear case, is that the designer must define the number (dimensionality) $n_{d}$ and proper placement of the disturbances in the model, under the restriction $n_{d} \leqslant n_{y}$.

\subsection{The technique of state disturbance prediction}

The technique proposed in this section is, on one hand, a further development of the technique presented in Section 3 for the case with measured process state. On the other hand, it is a generalisation of the approach presented in [23], to the nonlinear case. It differs from the technique with the extended state presented in the previous section, as it does not use extension of the state.

Application of a state observer is necessary when the process state cannot be measured. For the process (10) modeled by (11), a simple and straightforward deterministic 
approach to the observation of the process state is to use the Extended Luenberger Observer (ELO) in the standard form

$$
\hat{x}(k)=f(\hat{x}(k-1), u(k-1))+\boldsymbol{L}[y(k-1)-g(\hat{x}(k-1))],
$$

or, better, in the current observer form (see Section 4.1):

$$
\hat{x}(k)=f(\hat{x}(k-1), u(k-1))+\boldsymbol{L}_{c}[y(k)-g(f(\hat{x}(k-1), u(k-1))],
$$

where $\hat{x}(k)$ is the state estimate and $\boldsymbol{L}$ or $\boldsymbol{L}_{c}$ is the observer gain matrix. Design and analysis of the ELO for nonlinear processes is not so easy as for linear systems, even in the case without a process-model mismatch, see, e.g., [2]. The main assumption is local observability of the model in the area of operation of the feedback control system, and the simplest method to design the gain matrix is to do it as for a linear Luenberger observer, for a linearization of the model (11).

The analysis of nonlinear observers is out of scope of this paper, we shall further assume that the observer can be successfully designed (in the next section, design details will be given for exemplary processes). Any kind of a more elaborate observer, providing asymptotically stable estimation error in a working region of interest, can be also applied. Extended Kalman filter may be a sound choice, especially when the process state and outputs are under influence of noises.

The main appealing feature of the proposed nonlinear MPC algorithm is that the process state only is estimated by the observer (the process state is not augmented), despite modeling errors and external disturbances. The algorithm will be denoted Algorithm NMPC2. Like NMPC1, it solves at each sampling instant $k$ the nonlinear MPC optimization problem (8), by a nonlinear optimization procedure iterating the vector of decision variables $U(k)$ defined by (2), starting from an initial vector $U^{(0)}(k)$. For each new value $U^{(i)}(k)$ calculation of the output predictions $y^{(i)}(k+p \mid k)$ over the control horizon, $p=1, \ldots, N$, is performed.

\section{Algorithm NMPC2}

1. The process outputs $y(k)$ are measured.

2. The state disturbance prediction $v(k)$ is calculated:

$$
v(k)=\hat{x}(k)-f(\hat{x}(k-1), u(k-1)) .
$$

3. The optimization problem (8) is solved, iteratively by a nonlinear optimization procedure, which calculates at its every $(i$-th) internal iteration the output predictions in the following way:

3a. Using the model (12), state predictions $x^{(i)}(k+p \mid k)$ are recursively calculated over the control horizon (for $p=1, \ldots, N$ ):

$$
x^{(i)}(k+p+1 \mid k)=f\left(x^{(i)}(k+p \mid k), u^{(i)}(k+p \mid k)\right)+v(k),
$$


where $x^{(i)}(k \mid k)=\hat{x}(k)$ is the estimated state, and

$$
u^{(i)}(k+p \mid k)=u^{(i)}\left(k+N_{u}-1 \mid k\right), \text { for } p \geqslant N_{u} .
$$

3b. The output predictions are calculated:

$$
y^{(i)}(k+p \mid k)=g\left(x^{(i)}(k+p \mid k)\right)+[y(k)-g(\hat{x}(k))], \quad p=1, \ldots, N .
$$

4. After the optimal trajectory $\hat{U}(k)$ is found by the optimization procedure, its first element $\hat{u}(k \mid k)$ defines the current control input signal $u(k)=\hat{u}(k \mid k)$, which is sent to the process actuators.

5. The next process state estimate $\hat{x}(k+1)$ is calculated by the nonlinear observer, e.g., (33).

It should be noted that state predictions (35) incorporate the state disturbance prediction $v(k)$ and the output predictions are calculated, in step (3b), by a non-standard formula (37) which includes the newly introduced correction term $y(k)-g(\hat{x}(k))$ (compare with (17)).

The following Theorem 8 formulates conditions of offset-free property of the feedback control system with the Algorithm NMPC2.

\section{Theorem 8 Assume that}

- Assumptions 1 and 2 are satisfied,

- Assumption 4: The MPC optimization problem (8) with output predictions as defined by (35)-(37) in the NMPC2 algorithm, is feasible for every $k$ (i.e., the set defined by the constraints (3)-(5) is not empty) and the feedback control system consisting of the process (10a)-(10b) and the Algorithm NMPC2 (including the process state observer) is asymptotically stable.

Then the feedback control system defined in Assumption 4 provides offset-free control, i.e., the process outputs stabilize at the set-point values $y_{s s}^{s p}$, despite the influence of the disturbances defined in Assumption 1.

Proof. The first part of the proof is analogous to the first part of the proof of Theorem 1. The state prediction equations are analogous to (18a)-(18e), only state estimate $\hat{x}(k)$ must be used instead of the measured state $x(k)$.

The reasoning about the steady-state is also the same, only the steady-state value of the state estimate $\hat{x}_{s s}$ must be additionally taken into account, with $\hat{x}_{s s} \neq x_{s s}$, in general. As the state estimate $\hat{x}_{s s}$ is used now in the prediction equations in place of the measured state $x(k)$, then further reasoning as in Theorem 1 leads to the conclusion that all predictions $x(k+p \mid k)$ on the prediction horizon stabilize at the value $\hat{x}_{s s}$.

Taking now into account this result and the output prediction equation (37), we conclude that the predicted outputs stabilize, at the steady-state, at the value:

$$
y(k+p \mid k)=g\left(\hat{x}_{s s}\right)+\left[y_{s s}-g\left(\hat{x}_{s s}\right)\right]=y_{s s}, \quad p=1, \ldots, N .
$$


Therefore, due to the additional correction term $\left[y_{s s}-g\left(\hat{x}_{s s}\right)\right]$ in the prediction equation (37) in NMPC2 algorithm, the obtained result (38) is the same as in the NMPC1 algorithm, see (19). Therefore, the last part of the proof, i.e., proving that $y(k+p \mid k)=y_{s s}=$ $y_{s s}^{s p}, \quad p=1, \ldots, N$, is the same as in the proof of Theorem 1 . This completes the proof.

The key difference between the algorithm with measured state (Algorithm NMPC1) and the Algorithm NMPC2 is the introduction of the correction term $y_{s s}-g\left(\hat{x}_{s s}\right)$ in the final prediction equation (19). Without this term, the process outputs would stabilize at the value $y_{s s}=g\left(\hat{x}_{s s}\right)$, in general not equal to the value $y_{s s}^{s p}$, as in general $\hat{x}_{s s} \neq x_{x x}$. This is due to the influence of deterministic disturbances and is true also in the linear case. The reason is that at a steady-state the process equations $x_{s s}=f_{p}\left(x_{s s}, u_{s s}, d_{s s}\right)$ and $y_{s s}=g\left(x_{s s}\right)$ must be fulfilled, with results in the values $\left(x_{s s}, u_{s s}\right)$. On the other hand, the steady-state estimate value $\hat{x}_{s s}$ is a fixed point of the observer equation (for the obtained values $x_{s s}$ and $u_{s s}$ )

$$
\hat{x}_{s s}=f\left(\hat{x}_{s s}, u_{s s}\right)+\boldsymbol{K}\left(y_{s s}-g\left(\hat{x}_{s s}\right)\right)
$$

which is not equal to $x_{s s}$, in general. The reason is that if $f_{p}\left(x_{s s}, u_{s s}, d_{s s}\right) \neq f\left(\hat{x}_{s s}, u_{s s}\right)$, then we have in steady-state a non-zero correction term in the observer equation, because $y_{s s}-g\left(\hat{x}_{s s}\right) \neq 0$. This occurs also in the conventional approach with the extended process-and-disturbance state estimation recalled briefly in Section 4.1, due to differences in numbers and locations of disturbances in the process equations and estimated disturbances placed by the designer in the model equations (e.g., see results of simulations presented in [12]).

Comparing the main differences between the proposed technique (Algorithms NMPC1 and NMPC2) and the more conventional approach with the extended processand-disturbance state estimation [12], the advantage of the proposed one is an easier design, without the necessity of a careful choice and placement of estimated deterministic disturbances in the model (under the restriction $n_{d} \leqslant n_{y}$ !). Another advantage is a simpler controller structure, with the observer of the process state only (not augmented). On the other hand, it could be seen in certain cases as a disadvantage that the Algorithm NMPC2 applies to output control only, i.e., with the main part of the MPC performance function being the sum of squared output control errors (see (1)) - not squared differences between current and desired (reference) states of the process, as in [12]. This is due to the fact that only the outputs are measured, thus the correction term can be for the outputs only. Certainly, this restriction does not apply to the control structure with the Algorithm NMPC1, as the states are measured there and thus the correction term is not needed. 


\section{Simulation examples}

Example 1. A simple nonlinear process with 2-step delay, given be the equations:

$$
\begin{aligned}
x_{p 1}(k+1) & =0.32 x_{p 1}(k)+0.08 x_{p 1}(k)^{2}+0.2 x_{p 3}(k)+0.25 x_{p 4}(k)+0.02 x_{p 1}(k) x_{p 3}(k), \\
x_{p 2}(k+1) & =u(k)+d(k), \\
x_{p 3}(k+1) & =x_{p 2}(k), \\
x_{p 4}(k+1) & =x_{p 3}(k), \\
y(k) & =x_{p 1}(k),
\end{aligned}
$$

is considered, where $x_{p}(k)=\left[x_{p 1}(k) \cdots x_{p 4}(k)\right]^{T}$ is the process state and $d(k)$ the unknown (unmeasured) disturbance.

The following nonlinear model of the process is used for the controller design:

$$
\begin{aligned}
x_{1}(k+1) & =0.27 x_{1}(k)+0.1 x_{1}(k)^{2}+0.2 x_{3}(k)+0.25 x_{4}(k), \\
x_{2}(k+1) & =u(k), \\
x_{3}(k+1) & =x_{2}(k), \\
x_{4}(k+1) & =x_{3}(k), \\
y(k) & =x_{1}(k) .
\end{aligned}
$$

where $x(k)=\left[x_{1}(k) \cdots x_{4}(k)\right]^{T}$ is the model state. Notice differences in the coefficients of both models and presence of the unmodelled dynamics (last term in (40a)).

The MPC controllers were designed implementing NMPC 1 and NMPC 2 algorithms. The appropriate prediction horizon was found to be $N=8$, and the control horizon was set to $N_{u}=3$. Only one scalar weighting coefficient $\lambda=0.2$ was assumed in the performance function. The Extended Luenberger Observer (ELO, in current form) was used for state estimation. Due to the structure of the model, only one coefficient of the observer gain matrix needed to be designed, $L_{c}=\left[\begin{array}{llll}l_{1} & 0 & 0 & 0\end{array}\right]^{T}$, the value of $l_{1}=0.5$ was found experimentally. Initial state of the observer was $x_{o b s}\left(\begin{array}{ll}0 \\ )\end{array}=\left[\begin{array}{llll}0.5 & 0 & 0 & 0\end{array}\right]^{T}\right.$, whereas the initial process state was zero. Simulation results are shown in Fig. 1 for the NMPC1 and in Fig. 2 for the NMPC2 algorithms, respectively. The simulation scenario comprises unit steps of the set point at times $k=0$ and $k=20$ and a unit step of the input disturbance at time $k=40$. Both algorithms respond well and similarly to set-point changes, but NMPC1 attenuates the disturbance much better. This is due to the use of the state disturbance prediction technique, which enables quicker reaction in the case of a delay and measured state.

Example 2. A nonlinear SISO process with the state-space description is considered:

$$
\begin{aligned}
x_{p 1}(k+1) & =0.95 x_{p 1}(k)-0.25 x_{p 1}(k) x_{p 2}(k)+x_{p 2}(k)+d_{1}(k), \\
x_{p 2}(k+1) & =0.7 x_{p 2}(k)+0.1 x_{p 2}(k) d_{2}(k)+u(k), \\
y_{p}(k) & =x_{p 1}(k)+d_{3}(k),
\end{aligned}
$$



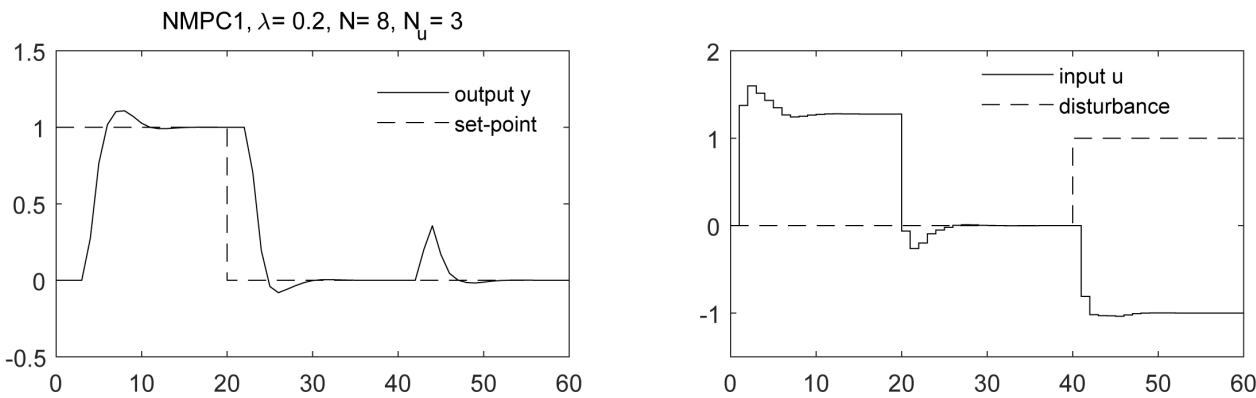

Figure 1: Algorithm NMPC1: trajectories of the set-point and measured output (left), process input and unmeasured input disturbance (right).
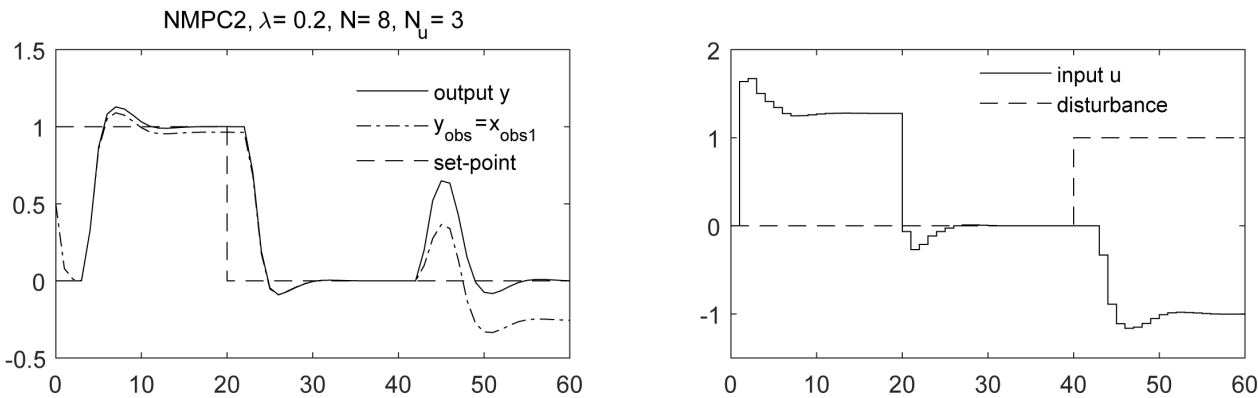

Figure 2: Algorithm NMPC2: trajectories of the set-point, measured and estimated outputs (left), process input and unmeasured input disturbance (right).

where $x_{p}(k)=\left[x_{p 1}(k) x_{p 2}(k)\right]^{T}$ is the system state, $d_{1}(k), d_{2}(k)$ are unknown (unmeasured) disturbances entering the state equations and $d_{3}(k)$ is an unknown (unmeasured) output disturbance. The presented example process is taken from [12], but it is with reacher structure of disturbances which can be mutually independent, whereas in [12] $d_{1}(k)=d_{2}(k)=d_{3}(k)=d(k)$ was assumed, i.e., one disturbing signal.

The following nonlinear model of the process is used for the controller design:

$$
\begin{aligned}
x_{1}(k+1) & =0.9 x_{1}(k)-0.3 x_{1}(k) x_{2}(k)+x_{2}(k), \\
x_{2}(k+1) & =0.8 x_{2}(k)+u(k), \\
y(k) & =x_{1}(k),
\end{aligned}
$$

where $x(k)=\left[x_{1}(k) x_{2}(k)\right]^{T}, y(k)$ are the model state and output, respectively. Notice differences in the coefficients of both models and presence of the unmodeled dynamics (last term in (42b)).

The MPC controller was designed implementing the algorithms NMPC1 and NMPC2. The appropriate prediction horizon was found to be $N=8$, and the control horizon was set to $N_{u}=4$. Only one scalar weighting coefficient $\lambda=0.5$ was assumed in the performance function, i.e., $\boldsymbol{\Psi}=\boldsymbol{I}$ and $\boldsymbol{\Lambda}=\lambda \boldsymbol{I}$ in (1). The simulation scenario was the same for both algorithms, comprising changes of the set-point and all disturbances. 
A representative result for the NMPC1 algorithm is shown in Fig. 3. The results show good performance of the algorithm for the considered example. Both the modeling inaccuracies (compare coefficients and structures of (42a)-(42c) and (43a)-(43c)) and step changes of all different and unknown (unmeasured) external disturbances are well attenuated, providing offset-free control.
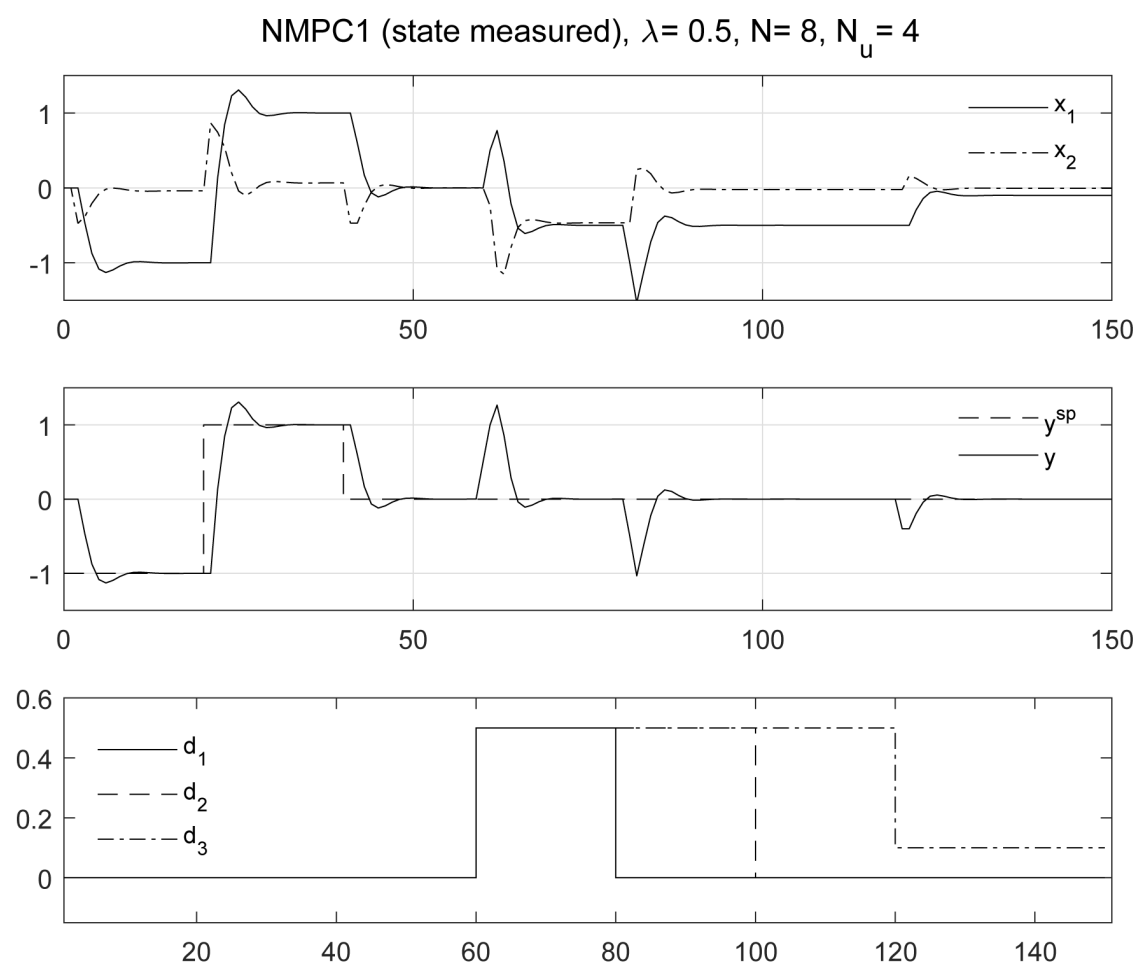

Figure 3: Algorithm NMPC1: trajectories of the measured states (top), set-point and process output (middle), unmeasured disturbances (bottom).

For the NMPC2 algorithm, the ELO observer in the current form, with constant gains, was found to be satisfactory. The gain matrix of the observer $\boldsymbol{L}_{c}$ was designed for the linear state-space model being linearization of the nonlinear model (43) at the origin, in a standard way, for a predefined set of eigenvalues for the closed loop (0.3 and 0.4), see, e.g., [1].

A representative result for the NMPC2 algorithm is shown in Fig. 4. The results show again good performance. When comparing with the NMPC1 results, similar quality of set-point tracking is observed. Attenuation of disturbances is worse, but not significantly.

For a comparison, the MPC nonlinear controller designed according to conventional technique of extended process-and-disturbance state estimation (see Section 4.1) was also tested (algorithms NMPCA). Since the example system is a SISO one, this design has to be under the restriction $\operatorname{dim} d(k)=n_{d}=1$. Constraining the design to single addi- 
NMPC2 (process observer), $L_{c}=[0.83,0.25], \lambda=0.5, N=8, N_{u}=4$
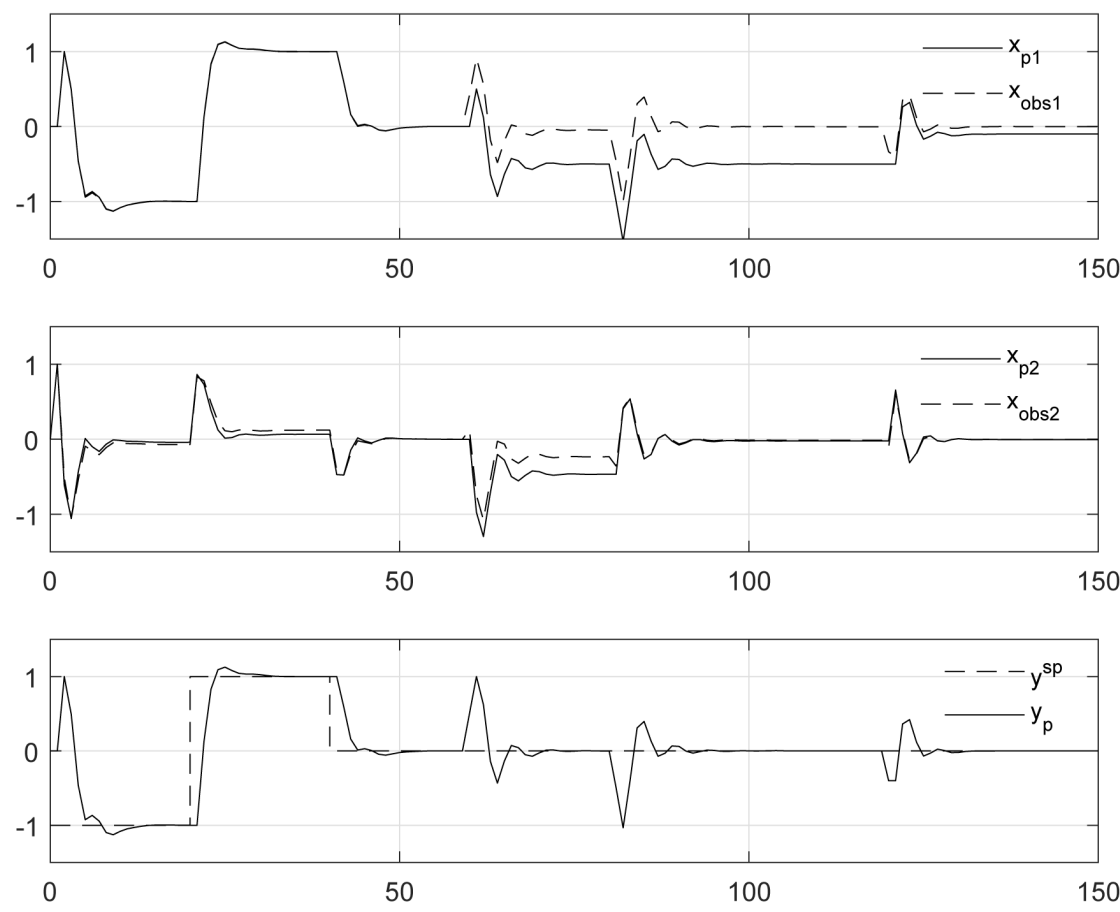

Figure 4: Algorithm NMPC2: trajectories of measured and observed states $x_{1}$ and $x_{2}$ (top and middle), set-point and process output (bottom); unmeasured disturbances as in Fig. 3.

tive disturbance $d(k)$, there are three possibilities for placing it in the model equations: as a disturbance of the first state (43a), of the second state (43b) or of the output equation (43c). All cases were tested. Results shown in Figures 3 and 4 suggest that the disturbance $d_{1}(k)$ is most crucial. Therefore, the following augmented state model was first tested:

$$
\begin{aligned}
x_{1}(k+1) & =0.9 x_{1}(k)-0.3 x_{1}(k) x_{2}(k)+x_{2}(k)+d(k), \\
x_{2}(k+1) & =0.8 x_{2}(k)+u(k), \\
d(k+1) & =d(k), \\
y(k) & =x_{1}(k),
\end{aligned}
$$

and the extended state vector was $[x(k) d(k)]^{T}$. This choice occurred to be not successful. First, it occurred very difficult to design the ELO observer. Second, the obtained simulation results were much worse, an example of best results is shown in Fig. 5.

Next, the disturbance $d(k)$ was placed in the second model equation, i.e., was located in (44b) instead of in (44a). This case occurred to be successful (the case of input disturbance), the simulation results obtained were similar to these with the NMPC2 algorithm 

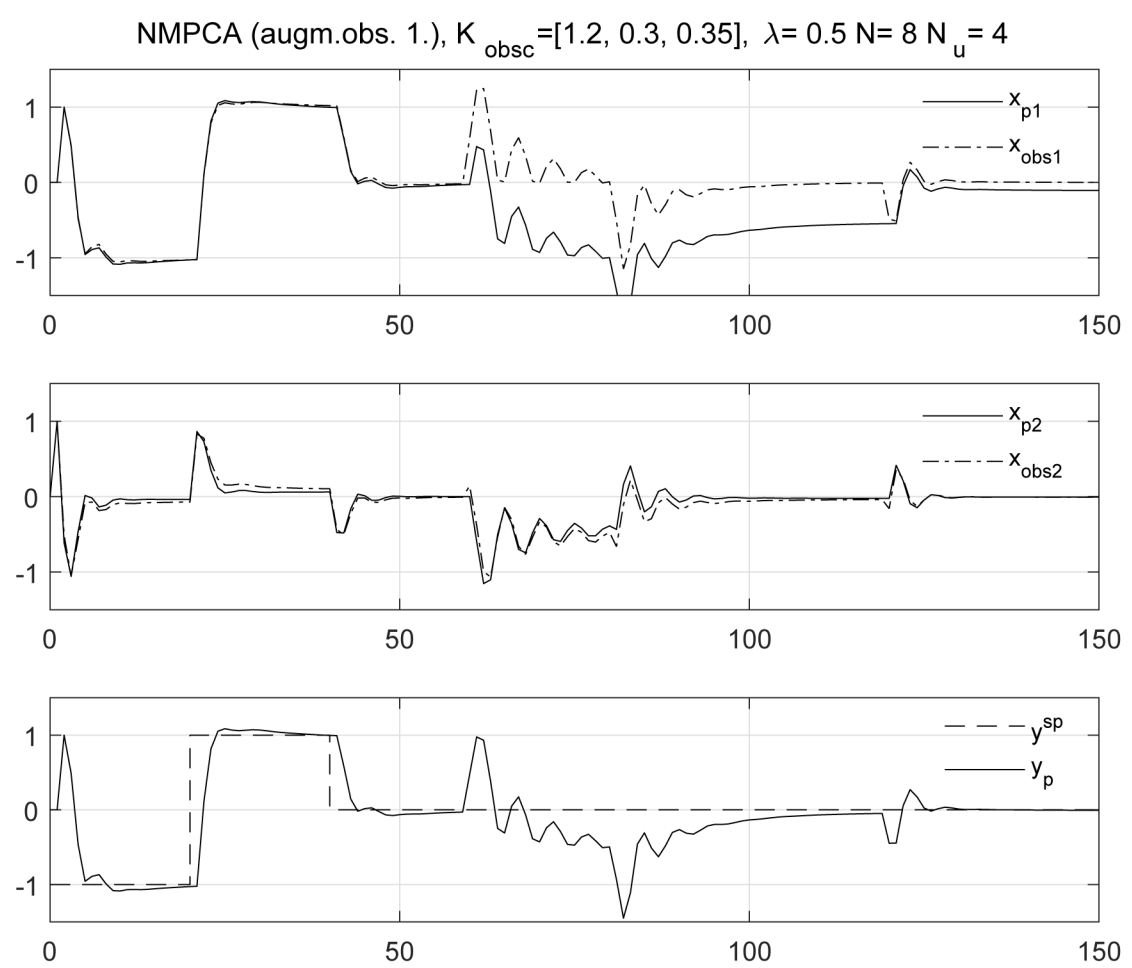

Figure 5: Algorithm NMPCA for the disturbance added to the first model equation: trajectories of measured and observed states $x_{1}$ and $x_{2}$ (top and middle), set-point and process output (bottom); unmeasured disturbances as in Fig. 3.

(not better). Since also in this case there is significant difference between disturbance structure in the process (42) and its model, trajectories of the state estimates significantly differed from the true state trajectories, similarly as it was for the NMPC 2 algorithm (see Fig. 4).

The third tested case was with the disturbance $d(k)$ in the output equation. This trial choice was a complete failure for the considered example, the ELO observer was not able to estimate the extended state in a way sufficient for a proper controller action.

The simulations with the extended Kalman filter (EKF) instead the ELO were also performed, for all cases presented above with the ELO. As the EKF uses gain matrix adaptation, better results could be awaited. For the cases with augmented process-anddisturbance state the results were generally better, but not better than with the NMPC2 algorithm. Again, the case with output disturbance was a complete failure.

Certainly, the presented comparison is for the chosen examples only. Nevertheless, it demonstrates that is is much simpler to design the control system using the proposed technique of state disturbance prediction than the more conventional technique of extended process-and-disturbance state and that the obtained results are competitive. 


\section{Conclusions}

The approach to offset-free model predictive control with nonlinear state-space process models based on the technique of state disturbance prediction has been presented in the paper, for deterministic asymptotically constant unmeasured external and internal disturbances (modeling errors) and asymptotically constant set-points (reference values). The presented control structure is derived for the output control, i.e. for MPC formulations with squared output control errors being the main part of the performance function. The presented approach was originally proposed by the author for linear state-space process models. In the paper, the formulation for nonlinear models is derived. The basic element is an appropriate formulation of state disturbances in the model used for the state and output prediction, called the constant state disturbance prediction. This leads to simpler control structures, in cases with measured state without the need of disturbance observer/filter at all. In other cases, it is sufficient to estimate the process state only, as opposed to the conventional approach with a process-and-disturbance state estimation. Due to the prescribed simple disturbance modeling, in both cases the design is simpler as it leads to the state observer/filter of lower order. Moreover, necessity of choosing a limited number of disturbances and their placement in the process model is avoided. Further, for cases with input delays, better disturbance attenuation can be achieved when using design with full state measurement. Theoretical analysis of the offset-free property of the proposed algorithms is provided, under the applicability conditions that are weaker than in the conventional approach.

The presented approach uses the formulation of the MPC performance function based on predicted control errors and process input increments, whereas in the approach discussed in [12], process state and input deviations from appropriate steady-state targets are used. However, the approach with predicted control errors seems to be most practical.

Theoretical results presented in the paper are illustrated by simulations of control structures with nonlinear processes. Comparing the presented theoretical and simulation results with those for the technique with extended process-and-disturbance state estimation, the conclusion must be drawn that the proposed approach is simpler and yields competitive results, thus significantly broadening the range of possible solutions available for to design engineer.

\section{References}

[1] K.J. Astrom and B. Wittenmark: Computer Controlled Systems. Prentice Hall, Upper Saddle River, 1997.

[2] J. BIRK and M. ZEITZ: Extended Luenberger observer for non-linear multivariable systems. Int. J. of Control, 47(6), (1988), 1823-1835. 
[3] T. L. Blevins, G. K. McMillan, W. K. Wojsznis and M. W. Brown: Advanced Control Unleashed. The ISA Society, Research Triangle Park, NC, 2003.

[4] T. L. Blevins, W. K. Wojsznis and M. NiXON: Advanced Control Foundation. The ISA Society, Research Triangle Park, NC, 2013.

[5] E.F. CAmacho and C. Bordons: Model Predictive Control. Springer Verlag, London, 1999.

[6] A. H. Gonzalez, E. J. Adam and J. L. Marchetti: Conditions for offset elimination in state space receding horizon controllers: A tutorial analysis. Chemical Engineering and Processing, 47 (2008), 2184-2194.

[7] M. ŁAWryńCZUK: Computationally Efficient Model Predictive Control Algorithms: A Neural Network Approach, Studies in Systems, Decision and Control, 3. Springer Verlag, Heidelberg, 2014.

[8] M. ŁAWRYŃCZUK: Nonlinear state-space predictive control with on-line linearisation and state estimation. Int. J. of Applied Mathematics and Computer Science, 25(4), (2015), 833-847.

[9] J.M. Maciejowski: Predictive Control. Prentice Hall, Harlow, England, 2002.

[10] U. MAEDER, F. BORELli and M. MORARI: Linear offset-free model predictive control. Automatica, 45 (2009), 2214-2222.

[11] U. MAEDER and M. MORARI: Offset-free reference tracking with model predictive control. Automatica, 46 (2010), 1469-1476.

[12] M. Morari and U. MAEDER: Nonlinear offset-free model predictive control. Automatica, 48 (2012), 2059-2067.

[13] K.R. Muske and T.A. BAdGwEll: Disturbance modeling for offset-free linear model predictive control. J. of Process Control, 12 (2002), 617-632.

[14] G. PAnnocchia and A. Bemporad: Combined design of disturbance model and observer for offset-free model predictive control. IEEE Trans. on Automatic Control, 52(6), (2007), 1048-1053.

[15] G. PANnOCCHia and J.B. RaWlings: Disturbance models for offset-free model predictive control. AIChE J., 49(2), (2003), 426-437.

[16] S.J. QIN and T.A. BADGWELL: A survey of industrial model predictive control technology. Control Engineering Practice, 11 (2003), 733-764.

[17] V. RAO and J. B. RAWlingS: Steady states and constraints in model predictive control. AIChE J., 45(6), (1999), 1266-1278. 
[18] J. B. Rawlings and D. Q. Mayne: Model Predictive Control: Theory and Design. Nob Hill Publishing, Madison, 2009.

[19] J.A. Rossiter: Model-Based Predictive Control. CRC Press, Boca Raton - London - New York - Washington,D.C., 2003.

[20] P. Tatjewski: Advanced Control of Industrial Processes. Springer Verlag, London, 2007.

[21] P. TATJEWSKI: Advanced control and on-line process optimization in multilayer structures. Annual Reviews in Control, 32 (2008), 71-85.

[22] P. TATJEWSKi: Supervisory predictive control and on-line set-point optimization. Int. J. of Applied Mathematics and Computer Science, 20(3), (2010), 483-496.

[23] P. TATJEWSKI: Disturbance modeling and state estimation for offset-free predictive control with state-spaced process models. Int. Journal of Applied Mathematics and Computer Science, 24(2), (2014), 313-323.

[24] P. TATJEWSKI: Offset-free nonlinear predictive control with measured state and unknown asymptotically constant disturbances. In K. Malinowski, J. Józefczyk, and J. Światek, (Eds) Aktualne problemy automatyki i robotyki (Actual problems in automation and robotics), pages 288-299. Akademicka Oficyna Wydawnicza (Academic Publisher) EXIT, Warszawa, 2014.

[25] P. TATJEWSKI: Sterowanie zaawansowane procesów przemystowych (Advanced Control of Industrial Processes), Second, revised edition (e-book, in Polish). Akademicka Oficyna Wydawnicza (Academic Publisher) EXIT, Warszawa, 2016.

[26] P. TATJEWSKi: Offset-free nonlinear model predictive control. In W. Mitkowski, J. Kacprzyk, K. Oprzedkiewicz, and P. Skruch, (Eds), Trends in Advanced Intelligent Control, Optimization and Automation, Proc. of 19th Polish Control Conference, Advances in Intelligent Systems and Computing 577, pages 33-44. Springer, 2017.

[27] P. TATJEWSKI and M. ŁaWRYŃCZUK: Soft computing in model-based predictive control. Int. J. of Applied Mathematics and Computer Science, 16(1), (2006), 7-26.

[28] L. WANG: Model Predictive Control System Design and Implementation using MATLAB. Springer Verlag, London, 2009. 